Itinéraires Itinéraires

Littérature, textes, cultures

2013-2 | 2014

Sade et les femmes

\title{
Noëlle Chatelet, Entretien avec le marquis de Sade
}

Paris, Plon, 2011, 145 pages.

\section{Anne Coudreuse}

\section{CpenEdition}

Journals

Édition électronique

URL : http://journals.openedition.org/itineraires/763

DOI : $10.4000 /$ itineraires.763

ISSN : 2427-920X

Éditeur

Pléiade

\section{Édition imprimée}

Date de publication : 1 mars 2014

Pagination : 204-207

ISBN : 978-2-343-02712-8

ISSN : 2100-1340

\section{Référence électronique}

Anne Coudreuse, « Noëlle Chatelet, Entretien avec le marquis de Sade», Itinéraires [En ligne], 2013-2 |

2014, mis en ligne le 01 novembre 2013, consulté le 22 septembre 2020. URL : http://

journals.openedition.org/itineraires/763; DOI : https://doi.org/10.4000/itineraires.763

Ce document a été généré automatiquement le 22 septembre 2020.

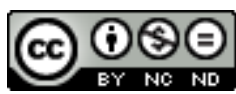

Itinéraires est mis à disposition selon les termes de la licence Creative Commons Attribution - Pas d'Utilisation Commerciale - Pas de Modification 4.0 International. 


\title{
Noëlle Chatelet, Entretien avec le marquis de Sade
}

Paris, Plon, 2011, 145 pages.

\author{
Anne Coudreuse
}

\section{RÉFÉRENCE}

Noëlle Chatelet, Entretien avec le marquis de Sade, Paris, Plon, 2011, 145 pages.

\section{NOTE DE L'ÉDITEUR}

Ce texte a été publié une première fois sur Nonfiction.fr.

\section{Un entretien semi-fictif}

Pour ce livre de commande, Noëlle Châtelet a imaginé une rencontre avec Sade, le vieillard obèse de soixante-treize ans, le 2 décembre 1813 , soit un an avant sa mort, à l'asile de Charenton où il était emprisonné. La situation de communication est fictive, mais les réponses du marquis aux questions de son interlocutrice sont toutes vraies, car elles sont extraites de ses livres et de ses écrits très nombreux, lettres, journal inédit, testament, et bien sûr ses fameux romans. Roland Barthes avait montré l'alternance dans les récits sadiens entre les "dissertations" et les scènes $d$ '" orgie ». Noëlle Châtelet n'a gardé pour ce montage de textes que les passages réflexifs, les textes argumentatifs, ou de nombreux extraits de lettres qui nous donnent une idée de la vie quotidienne du prisonnier Sade à la Bastille. Ce n'est donc pas à la pudeur que ce livre peut faire outrage, mais bien plutôt à la morale. Même si "lire Sade, dans son intégralité, tient d'une épreuve, d'un effort ", cela n'est pas "sans bénéfice ", car il participe à sa façon de "l'aventure intellectuelle des Lumières ». Il réfléchit, "grâce à des personnages et des situations emblématiques - en dépit de leur violence -, sur les 
grandes questions du siècle : le despotisme, la religion, la place de l'homme dans la nature et la matière, la relativité des lois, les méfaits possibles de la civilisation, la nécessité d'une remise en cause des morales individuelles et collectives ». Par des travaux plus anciens, Noëlle Châtelet a une très bonne connaissance des écrits sadiens. Elle a ainsi publié Système de l'agression en 1972, «choix et présentation des textes philosophiques et politiques de Sade ", et en 1979 l'introduction et les notes de Justine ou les Malheurs de la vertu pour la collection «Idées » chez Gallimard. Elle reprend la forme très prisée au XVIII ${ }^{e}$ siècle du dialogue philosophique pour donner à entendre «le romancier théoricien, l'analyste obsessionnel du plaisir", en laissant de côté ses "fantômes", dont elle n'ignore pas pourtant l'importance, comme le prouve cette citation d'une lettre écrite par Sade à sa femme en 1779, depuis le donjon de Vincennes: "Vous avez imaginé faire merveille en me réduisant à une abstinence atroce sur le péché de la chair. Eh bien, vous vous êtes trompés : vous avez échauffé ma tête, vous m'avez fait former des fantômes qu'il faudra que je réalise ».

\section{Prendre Sade au sérieux}

2 Dans la lignée de ce $\mathrm{xx}^{\mathrm{e}}$ siècle qui, selon la formule du titre de l'essai récent d'Éric Marty, a " pris Sade au sérieux » (Paris, Seuil, 2011), Noëlle Châtelet redonne à entendre cette voix qui ne s'est guère éteinte depuis les années 1950, sans l'étouffer sous les commentaires: "Dire aussi la frustration que constitue l'obligation d'être sa contemporaine, d'avoir à me situer le 2 décembre 1813, moi aussi, et pour cette raison même, de ne pouvoir lui conter, ici et là, ce qui s'est dit et fait ultérieurement autour de sa personne et de son œuvre (commentaires, mises en scène théâtrales, adaptations filmiques, etc.). En un mot, comment il a rebuté, autant qu'inspiré, les deux siècles qui l'ont suivi ! » L'entretien suit d'abord le fil de la biographie, après une sorte d'arrêt sur image sur la condition déplorable du détenu qui confère une certaine tonalité pathétique à ce début. À propos de ses parties de débauche, Sade se justifie ainsi : «Je suis libertin, je l'avoue ; j'ai conçu tout ce qu'on peut concevoir dans ce genre-là, mais je n'ai sûrement pas fait tout ce que j'ai conçu et ne le ferai sûrement jamais. Je suis un libertin, pas un criminel ni un meurtrier !» Pour trouver la référence de ce texte, il faut se reporter aux notes à la fin du volume : il s'agit d'une lettre à sa femme de juillet 1783 . Dans un siècle où Beccaria a beaucoup réfléchi aux délits et aux peines, Sade définit la prison comme " le poison le plus certain de l'âme », ce que notre moderne Observatoire des prisons ne viendrait sans doute pas contredire : «Il faudra que j'aille vivre dans un bois en sortant d'ici, par l'impossibilité où l'état dans lequel je suis me mettra de vivre avec des hommes! » (lettre à sa femme, 17 février 1779). Pour guérir "Monsieur le 6 », comme il s'appelle lui-même (en se donnant comme nom le numéro de sa cellule), il aurait fallu le «clôturer avec des filles »: "Monsieur le 6, au milieu d'un sérail, serait devenu l'ami des femmes; il aurait reconnu et senti que rien n'est plus beau, plus grand que le sexe et qu[e], hors le sexe, il n'est point de salut. [...] Tout cela aurait fini par le laisser dans l'apaisement; et voilà comme dans le sein du vice je l'aurais ramené à la vertu!» Soins homéopathiques en quelque sorte, qui lui valent cette réponse ironique de son interlocutrice : "Votre méthode thérapeutique ne manque pas de charme!» Citant la dédicace de La Philosophie dans le boudoir, cette "école de la lubricité », elle demande : «Pourquoi tant d'abominations ? [...] De quoi s'agit-il enfin ? [...] Quelle est la bonne distance pour vous lire? » La réponse est un extrait du roman Juliette ou les 
Prospérités du vice: "Pourquoi craindre de publier quand la vérité même arrache les secrets de la nature, à quelque point qu'en frémissent les hommes ? Le roman est aussi important que l'histoire au philosophe qui veut connaître l'homme, le saisir dans son intérieur ». Le marquis rappelle de manière très virulente son athéisme. Malgré son admiration pour Jean-Jacques Rousseau, il est loin d'admettre que « tous les hommes naissent égaux en droits et en force ». Dans l'optique du matérialisme absolu de Sade, la nature, identifiée à une mère par Rousseau, est une "marâtre, [une] force aveugle et imbécile ». Au prétexte que " la vertu n'est que relative » aux différents peuples et aux différents climats, Sade en vient à justifier l'inceste, ce que son interlocutrice ne peut laisser passer: "Vous tentez de m'embobiner! Votre brillante sophistique met la raison au service de la déraison! Encore une fois, dans votre œuvre, ce sont moins les descriptions de la lubricité et de la cruauté que l'on ne vous pardonne pas que leur rationalisation!» Reprenant la morale pessimiste de La Rochefoucauld, Sade décrit l'homme comme "vicieux dans le sein même de la vertu », ce qui va à contre-courant de toute la vogue sensible et morale $\mathrm{du}$ XVIII ${ }^{\mathrm{e}}$ siècle, très bien représentée par Greuze en peinture. C'est bien pourtant la rhétorique sensible et pathétique qu'il utilise dans les mots qu'il prête à la reine Marie-Antoinette avant sa mort sur l'échafaud le 16 octobre 1793.

\section{Un dialogue entre deux siècles : Sade, mon prophète}

Pour lui, « de toutes les lois, la plus affreuse est sans doute celle qui condamne à mort un homme ». Il manifeste donc une avance de deux siècles sur l'abolition de la peine de mort, et ce n'est pas là le moindre des paradoxes chez cet écrivain dont les romans sont remplis de crimes affreux. Autre paradoxe chez cet auteur de récits horribles où les femmes sont si maltraitées, il veut faire de la femme l'égale de l'homme dans la jouissance, à laquelle elle pourra se livrer « dans les maisons établies à ce sujet » : «De retour dans la société, elle y pourra parler aussi publiquement des plaisirs qu'elle aura goûtés qu'elle le fait aujourd'hui d'un bal ou d'une promenade ». N'annonce-t-il pas ainsi une certaine presse féminine actuelle et le goût moderne pour l'intime qui a envahi la scène publique?

On le voit, cet exercice de style cultivé dans l'entretien fictif n'est pas sans charmes ni sans intérêt, surtout quand l'interlocutrice sait comment poser les bonnes questions et où trouver des réponses qui relancent le débat. Il en reste une impression agréable d'une traversée de l'œuvre à grandes enjambées, et de retrouvailles avec des textes et un style qui apparente Sade à un écrivain talentueux, capable de passer d'une tonalité à l'autre, du registre des larmes à celui de l'ironie, de la réflexion sans fards à l'hypocrisie, comme quand il encense la vertu dans le Discours aux mânes de Marat et de Le Pelletier... Mais ce montage de textes a aussi ses limites, à cause en particulier d'un aspect disparate, et du mépris pour la chronologie et le contexte qu'il suppose. En cela, cet entretien se présente de fait comme une invitation à lire ou relire Sade, sans jamais prétendre avoir le dernier mot sur ce qu'Annie Lebrun appelait ce «bloc d'abîme »... 


\section{AUTEURS}

\section{ANNE COUDREUSE}

Université Paris 13, Sorbonne Paris Cité, Pléiade 